\title{
LOG KODAIRA DIMENSION OF HOMOGENEOUS VARIETIES
}

\author{
MICHEL BRION AND DE-QI ZHANG
}

\begin{abstract}
Let $V$ be a complex algebraic variety, homogeneous under the action of a complex algebraic group. We show that the $\log$ Kodaira dimension of $V$ is non-negative if and only if $V$ is a semi-abelian variety.
\end{abstract}

\section{INTRODUCTION}

Throughout this short note, we work over the field of complex numbers. Given a smooth quasi-projective variety $V$, we recall the definition of the log Kodaira dimension $\bar{\kappa}(V)$. Take any projective variety $X$ which contains $V$ as a dense open subset. By blowing up subvarieties of the (reduced) boundary $D:=X \backslash V$, we may assume that $X$ is smooth and $D$ is a simple normal crossing divisor. In this case, the pair $(X, D)$ is called a smooth projective compactification of $V$. Let $K_{X}$ be a canonical divisor of $X$. The $\log$ Kodaira dimension $\bar{\kappa}=\bar{\kappa}\left(X, K_{X}+D\right)$ is defined as the unique value

$$
\bar{\kappa} \in\{-\infty, 0,1, \ldots, \operatorname{dim} V\}
$$

such that for some positive constants $\alpha, \beta$, we have

$$
\alpha m^{\bar{\kappa}} \leq h^{0}\left(X, m\left(K_{X}+D\right)\right) \leq \beta m^{\bar{\kappa}}
$$

for any sufficiently large and divisible positive integer $m$. As $\bar{\kappa}$ is independent of the choice of the smooth projective compactification $(X, D)$ (cf. [4, $\S 11.1]$ ), we may set $\bar{\kappa}:=\bar{\kappa}(V)$.

Also, recall that a Cartier divisor $D$ on a projective variety $X$ is pseudo-effective if its numerical equivalence class is contained in the closure of the convex cone spanned by the effective divisor classes on $X$.

We may now state the main result of this note:

Theorem 1.1. Let $X$ be a smooth projective variety, and $G$ a connected algebraic group (possibly nonlinear) of automorphisms of $X$. Assume that $G$ has a dense open orbit $V$ in $X$, and $D:=X \backslash V$ is a simple normal crossing divisor. Then the following conditions are equivalent.

(1) The log canonical divisor $K_{X}+D$ is pseudo-effective.

2010 Mathematics Subject Classification. 14J50, 14L10, 14M17, 32M12.

Key words and phrases. log Kodaira dimension, homogeneous variety, semi-abelian variety.

The second-named author is supported by an ARF of NUS. 
(2) $K_{X}+D$ is linearly equivalent to 0 .

(3) The log Kodaira dimension $\bar{\kappa}(V)$ is non-negative.

(4) $G$ is a semi-abelian variety.

We recall that a semi-abelian variety is an algebraic group $G$ that lies in an exact sequence

$$
1 \longrightarrow T \longrightarrow G \longrightarrow A \longrightarrow 1
$$

where $T$ is an algebraic torus (isomorphic to a product of copies of the multiplicative group $\mathbb{G}_{m}$ ), and $A$ an abelian variety; then $G$ is connected and commutative.

Here are a few words about the proof of Theorem 1.1, which is very simple. If $V$ is not a semi-abelian variety, we show that the general member of some covering family of affine lines on $G$ is not contractible to a point in $V$. Chevalley's structure theorem for algebraic groups is also used, so our proof is quite geometric.

From Theorem 1.1, we derive the following purely group-theoretic statement:

Corollary 1.2. Let $G$ be a connected algebraic group, and $H \leq G$ a closed subgroup. Then $\bar{\kappa}(G / H) \leq 0$, with equality if and only if $H$ contains a closed normal subgroup $N$ of $G$ such that $G / N$ is a semi-abelian variety.

\section{Proofs of Theorem 1.1] And Corollary 1.2}

The following result is well-known. See [5, Lemma 5.11] for a generalization to singular pairs.

Lemma 2.1. Let $Y$ be a smooth projective variety and $A$ a simple normal crossing divisor on $Y$. Suppose that $C$ is the general member of a covering family of rational curves on $Y$. Let d be the number of times that $C$ meets $A$, i.e., the degree of the (reduced) support of the divisor $\nu^{*}\left(A_{\mid C}\right)$, where $\nu: \mathbb{P}^{1} \rightarrow C$ is the normalization. Then we have:

(1) If $d \leq 2$, then $\left(K_{Y}+A\right) \cdot C \leq 0$.

(2) If $d \leq 1$, then $\left(K_{Y}+A\right) \cdot C<0$. In particular $K_{Y}+A$ is not pseudo-effective.

We now prove Theorem 1.1.

$(2) \Rightarrow(3)$ and $(3) \Rightarrow(1)$ follow readily from the definitions of the log Kodaira dimension and pseudo-effectivity.

$(4) \Rightarrow(2)$. Since the semi-abelian variety $G \subseteq \operatorname{Aut}(X)$ is commutative and acts faithfully on $X$, the stabilizer of any point of $V$ is trivial, and hence $V \cong G$. Also, the action of the Lie algebra of $G$ on $X$ yields a trivialization of the log tangent bundle $T_{X}(-\log D)$ (see [8, Main Thm.] and [2, Thm. 2.5.1]). In particular, the determinant of $T_{X}(-\log D)$ is trivial as well, i.e., $K_{X}+D \sim 0$. 
$(1) \Rightarrow(4)$. Fix a point $x_{0} \in V$ and denote by $H \leq G$ its isotropy subgroup, so that $V$ is $G$-equivariantly isomorphic to the homogeneous space $G / H$, with both the $G$-actions from the left. Since $G$ acts faithfully on $V$, we have:

Claim 2.2. $H$ contains no non-trivial closed normal subgroup of $G$.

Next we show:

Claim 2.3. $H$ contains the image of every additive one-parameter subgroup of $G$.

We prove Claim 2.3. Assume the contrary that there exists an additive one-parameter subgroup of $G$, i.e., a homomorphism of algebraic groups $u: \mathbb{G}_{a} \rightarrow G$ (where $\mathbb{G}_{a}$ denotes the additive group), with image not contained in $H$. Then the $\mathbb{G}_{a}$-orbit $u\left(\mathbb{G}_{a}\right) \cdot x_{0}$ is a curve and isomorphic to $\mathbb{G}_{a} / F$, where the isotropy subgroup $F$ is a finite subgroup of $\mathbb{G}_{a}$ and hence is trivial. Thus we obtain an embedding

$$
\begin{aligned}
f: \mathbb{A}^{1} \cong \mathbb{G}_{a} & \cong u\left(\mathbb{G}_{a}\right) \cdot x_{0} \hookrightarrow G / H, \\
t & \longmapsto u(t) \cdot x
\end{aligned}
$$

and hence a rational curve $C \subset X$ which intersects the boundary $D$ at at most one point. Also, the translates of $C$ by $G$ form a covering family of rational curves on $X$. By Lemma 2.1, it follows that $K_{X}+D$ is not pseudo-effective, contradicting our assumption. This proves Claim 2.3 .

We return to the proof of Theorem 1.1. By Chevalley's structure theorem (see [7, Thm. 16]), $G$ lies in a unique extension

$$
1 \longrightarrow L \longrightarrow G \longrightarrow A \longrightarrow 1
$$

where $L$ is a connected linear algebraic group, and $A$ an abelian variety. Consider the unipotent radical $R_{u}(L)$; this is a closed connected normal subgroup of $G$. If $R_{u}(L)$ is non-trivial, consider the last non-trivial term of its lower central series, $U$. Then $U$ is a closed connected normal subgroup of $G$, isomorphic to the additive group of a finitedimensional vector space. Hence, $U$ is not contained in $H$ by Claim 2.2 ; but then $G$ has an additive one-parameter subgroup with image not contained in $H$, contradicting Claim 2.3. So $R_{u}(L)$ is trivial, i.e., $L$ is reductive. Thus, the derived subgroup $[L, L]$ is semi-simple, and hence generated by images of additive one-parameter subgroups. So $[L, L] \subseteq H$ by Claim 2.3 . As $[L, L]$ is a normal subgroup of $G$, Claim 2.2 implies that $[L, L]$ is trivial. So $L$ is an algebraic torus. Hence $G$ is a semi-abelian variety.

This completes the proof of Theorem 1.1. 


\section{Next we prove Corollary 1.2.}

Denote by $N$ the kernel of the action of $G$ on $G / H$, i.e., the largest (closed ) normal subgroup of $G$ contained in $H$. Replacing $G, H$ with $G / N, H / N$, respectively, we may assume that $G$ acts faithfully on $G / H$.

By [3, Thm. 2], there exists a projective compactification $X$ on $G / H$ such that the natural $G$-action on $G / H$ from the left extends to an algebraic action on $X$. Using equivariant resolution of singularities (see [6]), we may assume that $X$ is smooth and $X \backslash V$ is a simple normal crossing divisor. Then the desired assertion follows from Theorem 1.1. This proves Corollary 1.2.

\section{CONCLUding REMARKS}

3.1. With the assumptions of Theorem 1.1, the action of the Lie algebra of $G$ on $X$ preserves $D$, and hence yields a linear map

$$
\mathrm{op}_{X, D}: \operatorname{Lie}(G) \longrightarrow H^{0}\left(X, T_{X}(-\log D)\right)
$$

where $T_{X}(-\log D)$ denotes the log tangent bundle. Moreover, the pull-back of $T_{X}(-\log D)$ to $V$ is just the tangent bundle $T_{V}$, which is generated by the image of $\operatorname{op}_{X, D}$. It follows that the induced map

$$
\wedge^{n} \operatorname{Lie}(G) \longrightarrow H^{0}\left(X,-\left(K_{X}+D\right)\right)
$$

is nonzero, where $n:=\operatorname{dim}(V)=\operatorname{dim}(X)$. In particular, $-\left(K_{X}+D\right)$ is effective. Thus, the pseudo-effectivity of $K_{X}+D$ in Theorem 1.1 (1) immediately implies Theorem 1.1 (2): $K_{X}+D \sim 0$.

3.2. Theorem 1.1 is applicable to an arbitrary (not necessarily smooth) equivariant compactification $Y \supseteq V$ as long as $Y$ is normal, the boundary $E:=Y \backslash V$ is a divisor, and $K_{Y}+E$ is $\mathbb{Q}$-Cartier. Indeed, by equivariant resolution of singularities, there exists a $G$-equivariant birational morphism $f: X \rightarrow Y$ such that $X$ is smooth, projective and equipped with a $G$-action, and $D:=X \backslash V$ (which is the inverse of $E$ ) is a simple normal crossing divisor. By the logarithmic ramification divisor formula, we have

$$
K_{X}+D=f^{*}\left(K_{Y}+E\right)+R_{f}
$$

where $R_{f}$ is an effective $f$-exceptional divisor. Thus the pairs $(X, D)$ and $(Y, E)$ (as compactifications of $V$ ) have the same log Kodaira dimensions: $\kappa\left(X, K_{X}+D\right)=\kappa\left(Y, K_{Y}+E\right)$, and the log canonical divisor $K_{X}+D$ is pseudo-effective if and only if so is $K_{Y}+E$.

In particular, if $K_{Y}+E$ is pseudo-effective, then so is $K_{X}+D$, and hence both $V \subseteq X$ and $V \subseteq Y$ are the embeddings of the semi-abelian variety $V$ in $X$ and $Y$, respectively. 
3.3. The normal projective equivariant compactifications of a given semi-abelian variety are well-understood by work of Alexeev (see [1]), where they are called semi-abelic varieties. In particular, for a semi-abelian variety $G$ given by an extension

$$
1 \rightarrow T \rightarrow G \rightarrow A \rightarrow 1
$$

where $T$ is an algebraic torus and $A$ an abelian variety, and a normal projective equivariant compactification $Y$ of $G$, the morphism $q: G \rightarrow A$ extends to a $T$-invariant morphism

$$
f: Y \longrightarrow A,
$$

which is a fibration in projective toric varieties with the big torus $T$. Thus, $f$ is the Albanese map of the variety $Y$.

\section{REFERENCES}

[1] V. Alexeev, Complete moduli in the presence of semiabelian group actions, Ann. of Math. (2) 155 (2002), no. 3, 611-708.

[2] M. Brion, Log homogeneous varieties, Proceedings of the XVIth Latin American Algebra Colloquium, 1-39, Bibl. Rev. Mat. Iberoamericana, Madrid, 2007.

[3] M. Brion, Some basic results on actions of nonaffine algebraic groups, Symmetry and spaces, 1-20, Progr. Math. 278, Birkhäuser, Boston, 2010.

[4] S. Iitaka, Algebraic Geometry - an Introduction to Birational Geometry of Algebraic Varieties, Grad. Texts in Math. 76, Springer 1981.

[5] S. Keel and J. McKernan, Rational curves on quasi-projective surfaces, Mem. Amer. Math. Soc. 140 (1999), no. 669.

[6] J. Kollár, Lectures on resolution of singularities, Ann. of Math. Stud. 166, Princeton Univ. Press, 2007.

[7] M. Rosenlicht, Some basic theorems on algebraic groups, Amer. J. Math. 78 (1956), 401-443.

[8] J. Winkelmann, On manifolds with trivial logarithmic tangent bundle, Osaka J. Math. 41 (2004), no. $2,473-484$.

Institut Fourier, Université de Grenoble, B.P. 74, 38402 SAint-Martin D'Hères, France

E-mail address: Michel.Brion@ujf-grenoble.fr

Department of Mathematics, National University of Singapore,

10 Lower Kent Ridge Road, Singapore 119076

E-mail address: matzdq@nus.edu.sg 